University of Wollongong

Research Online

Faculty of Engineering and Information

Faculty of Engineering and Information

Sciences - Papers: Part A

Sciences

2005

Reinforcement of macroscopic carbon nanotube structures by polymer intercalation: The role of polymer molecular weight and chain conformation

C J. Frizzell

University of Texas, Dallas

Marc in het Panhuis

University of Texas, Dallas, panhuis@uow.edu.au

D H. Coutinho

University of Texas, Dallas

$\mathrm{K}$ J. Balkus

University of Texas, Dallas

A Minett

University of Wollongong, aminett@uow.edu.au

See next page for additional authors

Follow this and additional works at: https://ro.uow.edu.au/eispapers

Part of the Engineering Commons, and the Science and Technology Studies Commons

Research Online is the open access institutional repository for the University of Wollongong. For further information contact the UOW Library: research-pubs@uow.edu.au 


\title{
Reinforcement of macroscopic carbon nanotube structures by polymer intercalation: The role of polymer molecular weight and chain conformation
}

\author{
Abstract \\ Novel polymer nanotube composites were fabricated by intercalating poly(vinylpyrrolidone) into \\ Buckypaper from solution. This was carried out for both low $(10 \mathrm{~kg} / \mathrm{mol})$ and very high $(1.3 \mathrm{Mg} / \mathrm{mol})$ \\ molecular weight polymers. Measurements of the polymer mass uptake as a function of time allowed the \\ calculation of diffusion coefficients as $1.66 \times 10-9 \mathrm{~cm} 2 / \mathrm{s}$ and $3.08 \times 10-12 \mathrm{~cm} 2 / \mathrm{s}$ for the low and high \\ molecular weight strands, respectively. Taking into account the molecular weights, comparison of these \\ coefficients suggests that each polymer type undergoes a different mode of diffusion: normal diffusion \\ for the $10 \mathrm{~kg} / \mathrm{mol}$ polymer, but reptation for the $1.3 \mathrm{Mg} / \mathrm{mol}$ polymer. This means that while the low weight \\ polymer retains its randomly coiled conformation during diffusion and adsorption, the $1.3 \mathrm{Mg} / \mathrm{mol}$ \\ molecule is forced to adopt an extended, high entropy state. These differences are reflected in the \\ mechanical properties of the intercalated papers. While reinforcement was observed in all cases, \\ modulus (increase $\sim \times 3.5$ ) and strength (increase $\sim \times 6$ ) enhancement occurred at lower polymer content \\ for the longer chain polymer. However, the papers intercalated with the shorter chain molecules were \\ much tougher (increase $\sim \times 25$ ). This is consistent with the conformation scheme described above.

\section{Disciplines} \\ Engineering | Science and Technology Studies

\section{Publication Details} \\ Frizzell, C. J., in het Panhuis, M., Coutinho, D. H., Balkus, K. J., Minett, A. I., Blau, W. \& Coleman, J. (2005). \\ Reinforcement of macroscopic carbon nanotube structures by polymer intercalation: The role of polymer \\ molecular weight and chain conformation. Physical Review B (Condensed Matter and Materials Physics), \\ 72 (21 December), 245420-1-245420-8.
}

\section{Authors}

C J. Frizzell, Marc in het Panhuis, D H. Coutinho, K J. Balkus, A Minett, W J. Blau, and J N. Coleman 


\title{
Reinforcement of macroscopic carbon nanotube structures by polymer intercalation: The role of polymer molecular weight and chain conformation
}

\author{
C. J. Frizzell \\ Department of Physics \& NanoTech Institute, The University of Texas at Dallas, 2601 N. Floyd Rd., Richardson, Texas 75083, USA \\ and Department of Physics, Trinity College Dublin, Dublin 2, Ireland \\ M. in het Panhuis* \\ Department of Physics \& NanoTech Institute, The University of Texas at Dallas, 2601 N. Floyd Rd., Richardson, Texas 75083, USA \\ and Department of Physical Sciences, the University of Hull, Hull, HU6 7RX, United Kingdom \\ D. H. Coutinho and K. J. Balkus, Jr. \\ Department of Chemistry, The University of Texas at Dallas, 2601 N. Floyd Rd., Richardson, Texas 75083, USA
}

A. I. Minett, W. J. Blau, and J. N. Coleman

Department of Physics, Trinity College Dublin, Dublin 2, Ireland

(Received 1 August 2005; revised manuscript received 28 October 2005; published 21 December 2005)

\begin{abstract}
Novel polymer nanotube composites were fabricated by intercalating poly(vinylpyrrolidone) into Buckypaper from solution. This was carried out for both low $(10 \mathrm{~kg} / \mathrm{mol})$ and very high $(1.3 \mathrm{M} \mathrm{g} / \mathrm{mol})$ molecular weight polymers. Measurements of the polymer mass uptake as a function of time allowed the calculation of diffusion coefficients as $1.66 \times 10^{-9} \mathrm{~cm}^{2} / \mathrm{s}$ and $3.08 \times 10^{-12} \mathrm{~cm}^{2} / \mathrm{s}$ for the low and high molecular weight strands, respectively. Taking into account the molecular weights, comparison of these coefficients suggests that each polymer type undergoes a different mode of diffusion: normal diffusion for the $10 \mathrm{~kg} / \mathrm{mol}$ polymer, but reptation for the $1.3 \mathrm{Mg} / \mathrm{mol}$ polymer. This means that while the low weight polymer retains its randomly coiled conformation during diffusion and adsorption, the $1.3 \mathrm{M} \mathrm{g} / \mathrm{mol}$ molecule is forced to adopt an extended, high entropy state. These differences are reflected in the mechanical properties of the intercalated papers. While reinforcement was observed in all cases, modulus (increase $\sim \times 3.5$ ) and strength (increase $\sim \times 6$ ) enhancement occurred at lower polymer content for the longer chain polymer. However, the papers intercalated with the shorter chain molecules were much tougher (increase $\sim \times 25$ ). This is consistent with the conformation scheme described above.
\end{abstract}

DOI: 10.1103/PhysRevB.72.245420

PACS number(s): 81.07.De, 62.25.+g, 81.05.Qk

\section{INTRODUCTION}

Single-walled carbon nanotubes (SWNT) are a remarkable material with superb mechanical properties. Exceptional values of up to $1500 \mathrm{GPa}$ for Young's modulus ${ }^{1}$ and $\sim 50 \mathrm{GPa}$ for tensile strength $^{2}$ have been demonstrated. However, potential macroscale applications have been hindered as bulk nanotube material consists mainly of aggregated bundles bound together by weak van der Waals interactions. This results in a massive reduction in the bulk mechanical properties when compared to that of individual tubes. Recently, some progress has been made using polymer solution based processing to organize nanotube containing powder into functional macroscale composite materials in the form of fibers ${ }^{3}$ and films. ${ }^{3-5}$ However, these improvements in nanotube based material properties rely on dispersing nanotubes in a polymer based matrix.

In previous work ${ }^{6}$ it was shown that the reverse procedure of polymer intercalation can be used to reinforce bulk nanotube materials. The mechanical properties of as-prepared (pristine) sheets of carbon nanotubes (Buckypaper) were significantly improved by soaking the sheets in various organic polymer solutions. Increases of approximately $\times 3$ in Young's modulus and $\times 9$ in tensile strength were obtained.
This work is important as polymer intercalation (either in situ or postproduction) is required to maximize both the strength and toughness of high performance nanotube fibers. ${ }^{3}$ However, questions such as the intercalation mechanism and the role of molecular weight remained unanswered.

In this paper, we use carbon nanotube sheets (Buckypaper) as a model system to examine the reinforcement effects of polymer intercalation on macroscale nanotube structures. The effects of soaking Buckypaper in organic polymer solutions of poly(vinylpyrrolidone) (PVP) are investigated. Studies of polymer intercalation as a function of time for two molecular weights demonstrate the diffusion mechanisms for low and high molecular weight polymers. Mechanical studies show that reinforcement is significantly more efficient for the high molecular weight polymer. This is shown to be related to the polymer conformation during the diffusion and subsequent adsorption process.

\section{EXPERIMENTAL DETAILS}

Carbon nanotube sheets (Buckypaper) were made by dispersion of HiPco SWNT (Carbon Nanotechnologies, Inc., HiPco Bucky Pearls ${ }^{\mathrm{TM}}$ batch no. P0-185) ${ }^{7}$ in aqueous solutions of Triton X-100. Single-walled carbon nanotubes are 

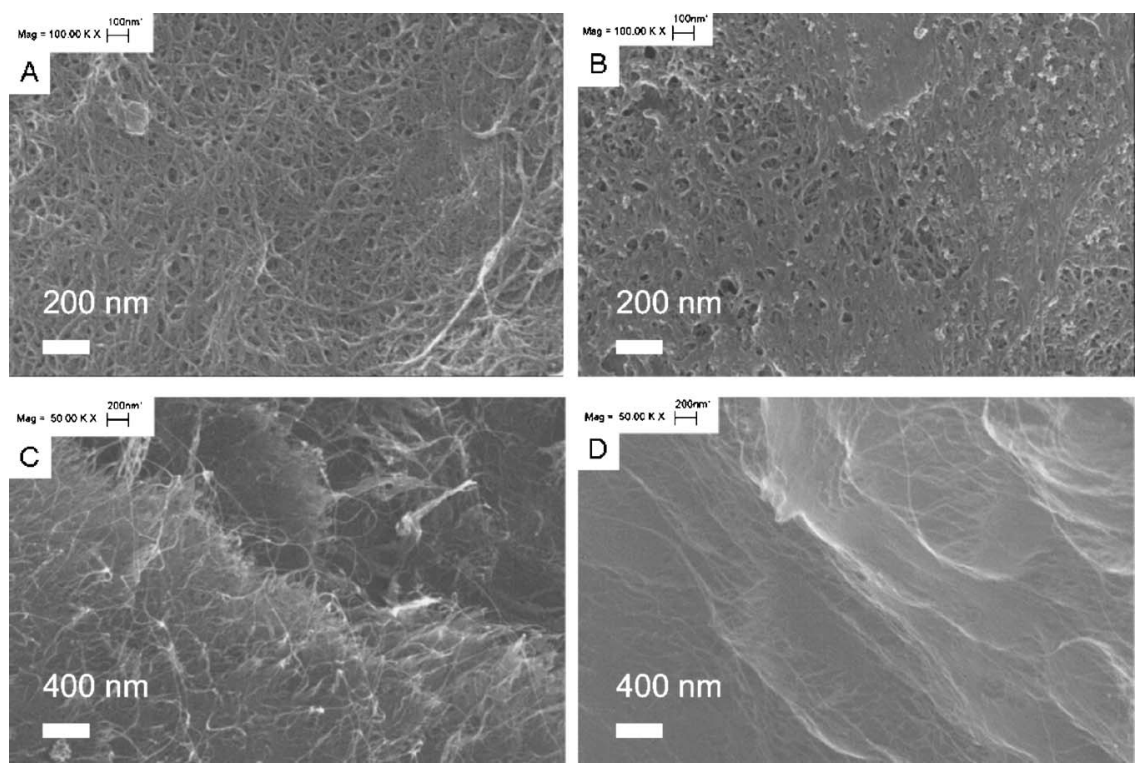

FIG. 1. Scanning electron micrograph images of typical reference (pristine) and polymer soaked Buckypaper. (a) Surface and (c) cross section of reference paper. (b) Surface and (d) cross section of paper soaked in PVP $(10000 \mathrm{~g} / \mathrm{mol})$ solution for $12 \mathrm{~h}$.

produced by the high pressure decomposition of carbon monoxide in the presence of an iron catalyst (indicated by the acronym HiPco). ${ }^{7}$ Dispersions were prepared by mixing $180 \mathrm{mg}$ of HiPco SWNT in $0.5 \%$ (wt/vol) aqueous solutions of Triton X-100, using a combination of low power (bath) and high power (tip) sonication. These dispersions were then vacuum filtered through Millipore Mitex PTFE membrane filters (pore size $=5 \mu \mathrm{m}$, diameter $=90 \mathrm{~mm}$ ). The resulting nanotube sheets were washed with $5 \mathrm{~L}$ water, followed by washing with ethanol until the foam disappeared, indicating that the majority of the surfactant had been removed. Subsequently, the sheets were allowed to dry under vacuum for $12 \mathrm{~h}$. The as-prepared (pristine) sheets were then cut into rectangular strips of $\approx 2 \mathrm{~mm} \times \approx 20 \mathrm{~mm} \times \approx 60 \mu \mathrm{m}$ and annealed at $500{ }^{\circ} \mathrm{C}$ for $2 \mathrm{~h}$ under flowing argon to remove any residual surfactant and contaminants. Density measurements were made for each strip using a Whitworth digital caliper for width and length measurement, a Mitoutoyo micrometer for thickness measurements and a Mettler Toledo microbalance for mass measurements.

Aqueous solutions of $2.5 \%$ (wt/vol) PVP (an amphiphilic semiconjugated polymer) in deionized water were prepared using polymer molecular weights $\left(M_{w}\right)$ of 10000 and $1300000 \mathrm{~g} / \mathrm{mol}$. A number of Buckypaper strips were soaked in polymer solutions of both molecular weights for various soak times. For each $M_{w}$ and soaktime, five strips were soaked. Soak times ranged from $10 \mathrm{~min}$ to $24 \mathrm{~h}$ for low molecular weight polymer and from 12 to $360 \mathrm{~h}$ for high molecular weight polymer. After soakage, all strips were carefully rinsed in deionized water and placed in a vacuum oven for $12 \mathrm{~h}$ at approximately $60{ }^{\circ} \mathrm{C}$ to remove residual water from the samples. Strip masses were measured a second time and any mass increase recorded. All reported mass increases are averages over five strips.

Tensile tests were carried out on all samples to determine Young's modulus, strength and toughness using a Perkin Elmer DMA7e. For all soak times, five strips were measured and the mean and standard deviation calculated. Scanning electron microscopy (SEM) images of various strips were taken using a Leo 1530VP field emission SEM. Thermogravimetric analysis (TGA) was carried out to determine polymer mass percentage and catalyst content in the sheets using a Perkin Elmer Pyris TGA1 under an oxygen atmosphere (scan rate $10 \mathrm{~K} / \mathrm{min}$ ).

Nitrogen absorption and desorption experiments were carried out on annealed sheets. The results were analyzed using multiple theories such as the BET, BJH, and HK methods. The specific surface area is calculated (using BET method) from information about the amount of $\mathrm{N}_{2}$ molecules absorbed on the surface and the surface area occupied by each molecule. The pore characteristics are calculated (using BJH and HK methods) from the fact that smaller pores will fill up quicker than the larger pores during absorption.

\section{RESULTS AND DISCUSSION}

After fabrication the Buckypaper strips resembled pieces of stiff matt black paper. Shown in Fig. 1(a) is an SEM image of the surface of a typical strip. It can be clearly seen that the surface of the material is made up of bundles of nanotubes with diameters ranging up to tens of nanometers. In addition a large number of pores can be seen indicating that the bundles pack together rather inefficiently. The pore sizes appear to be of the same order as the bundle diameters. Shown in Fig. 1(c) is an SEM image of a fracture surface allowing the investigation of the inside of the paper. It can be seen that the interior is characterized by substantial free volume again emphasizing the inefficient nature of the bundle packing. This is underlined by the low density of the material which was measured to be $\rho_{\text {paper }}=520 \pm 100 \mathrm{~kg} / \mathrm{m}^{3}$. The interbundle fractional free volume $\left(V_{f} / V_{T}\right)$ can be calculated from the density by $V_{f} / V_{T}=1-\rho_{\text {paper }} / \rho_{\mathrm{NT}}$, where $\rho_{\mathrm{NT}}$ is the nanotube bundle density which can be estimated as $1500 \mathrm{~kg} / \mathrm{m}^{3}$ (Ref. 6). This results in an interbundle free volume of $V_{f} / V_{T}=66 \pm 7 \%$ meaning that the material is dominated by the pores.

To investigate this further, $\mathrm{N}_{2}$ adsorption/desorption isotherms were measured for the annealed Bucky paper. This 


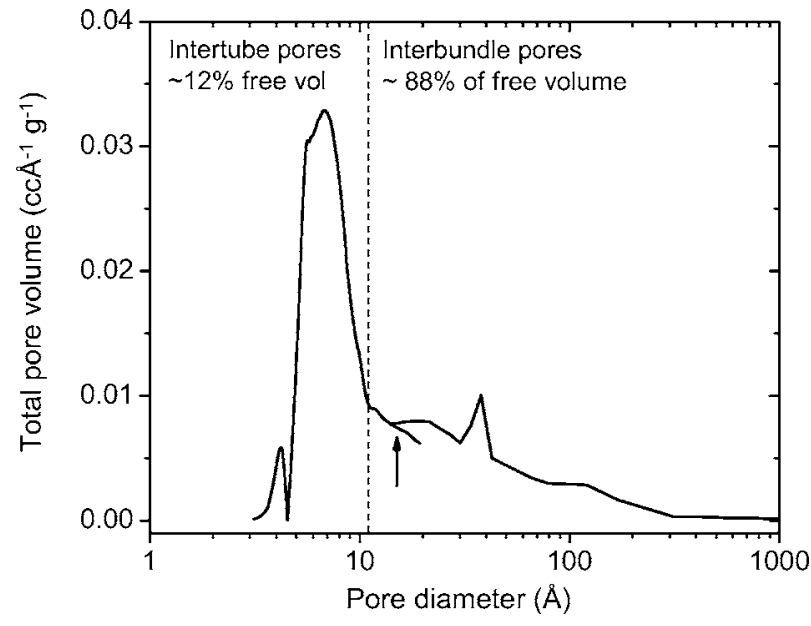

FIG. 2. Pore size distribution for annealed Buckypaper measured from $\mathrm{N}_{2}$ adsorption-desorption isotherms. Pore sizes of $<2 \mathrm{~nm}$ were calculated by the HK method while those of $>2 \mathrm{~nm}$ were calculated using the BJH method. The point where the data sets were joined is shown by the arrow where a small mismatch can be seen. The peak around $6 \AA$ is associated with internanotube pores; that is, the channels between nanotubes within bundles. The upper limit of this region is marked by the dotted line. The region of the graph to the right of the dotted line represents the interbundle pores.

data could then be used to calculate the pore size distribution within the sheet. This is carried out using the Horvath-Kawazoe $^{8}$ (HK) method to calculate small-diameter pore sizes $(<2 \mathrm{~nm})$ and the Barrett, Joyner, and Halenda (BJH) method $^{9}$ to calculate the size of larger pores $(>2 \mathrm{~nm})$. Both methods gave results that agreed well in the crossover region around $2 \mathrm{~nm}$. Both data sets have been combined and are shown in Fig. 2. The crossover region is indicated by the arrow. The data is dominated by a large peak between 4 and $11 \AA$. These relatively small pores are associated with the channels between nanotubes within bundles and can be thought of as interstitial pores. It should be noted that as the nanotubes are capped, the internal channels are not accessible to either gas adsorption or molecular intercalation. For trigonal packing within bundles, the interstitial pore size as a function of nanotube diameter can be easily calculated. ${ }^{10}$ This shows that the upper limit of the distribution of smaller pores shown in Fig. 1 occurs for bundles of nanotubes with diameter $\sim 1.3 \mathrm{~nm}$. This is close to the upper end of the diameter range for HiPco nanotubes. ${ }^{11}$ Numerical integration shows that these intertube pores contribute $\sim 12 \%$ of the total free volume of the Buckypaper. However, the pores with diameter larger than $11 \AA$ are associated with the space between bundles. It is these interbundle pores that are observed in the SEM images. The distribution displays peaks at approximately $4 \mathrm{~nm}$ and a long tail out to $100 \mathrm{~nm}$. As they contribute $\sim 88 \%$ of the total free volume, the presence of these pores will have a significant impact on the physical properties of the paper as a whole.

In addition, multipoint Brunauer, Emmett, and Teller ${ }^{12}$ (BET) analysis was used to calculate the specific surface area, $A_{S}$, of the paper. This was calculated as $A_{S}=611 \mathrm{~m}^{2} / \mathrm{g}$ which is slightly larger than previous reports. ${ }^{13}$ By neglecting the contribution due to the intertube pores $A_{S}$ can be used to approximate the bundle diameter. Assuming that $A_{S}$ is simply due to the outer surface of a large number of bundles with diameter, $D_{\text {bun }}$, then $A_{S}$ can be expressed as $A_{S}$ $=4 / \rho_{\mathrm{NT}} D_{\text {bun }}$, yielding $D_{\text {bun }}=4.5 \mathrm{~nm}$. However, this value is rather small and should be considered a lower limit.

In partial summary, the Buckypaper can be considered an amalgam of randomly ordered nanotube bundles with diameters ranging from a few nanometers to a few tens of nanometers. Between these loosely packed bundles is empty space which makes up $60-70 \%$ of the volume of the paper. This free volume consists of pores with sizes ranging from one to hundreds of nanometers. In short this material should be well suited for the intercalation of polymeric material.

In order to study the intercalation of polymers into the free volume discussed above, sheets of Buckypaper were soaked for various times in solutions of PVP of two different molecular weights. After soaking the sheets were rinsed and dried before weighing to measure polymer mass uptake. In all cases significant mass uptake was observed. To check that this did not just consist of a surface coating, SEM studies were carried out. Shown in Fig. 1(b) is an SEM image of a Buckypaper strip after soaking for $12 \mathrm{~h}$ on PVP with $M_{w}$ $=10000 \mathrm{~g} / \mathrm{mol}$. By comparison with Fig. 1(a) it can clearly be seen that a layer of polymer has coated the surface of the sheet. However, as the pores are still visible this layer cannot be very thick, suggesting that the majority of the mass uptake is associated with intercalated polymer. To confirm this, an SEM image was taken of a fracture surface allowing us to image the interior of the sheet. This is shown in Fig. 1(d) and clearly shows that the polymer has intercalated far into the bulk of the paper. In fact no regions were observed corresponding to Fig. 1(c) indicating that the intercalated polymer had covered the entire internal surface of the paper. Similar results were obtained for the $M_{w}=1.3 \mathrm{Mg} / \mathrm{mol} \mathrm{PVP} \mathrm{(not}$ shown). This shows clearly that the polymer macromolecules can diffuse very long distances into the paper before adsorbing onto the internal surface of the paper.

To understand the diffusion process the mass of intercalated polymer, termed the mass uptake, was measured for a number of soak times for each molecular weight. This is shown in Figs. 3(a) and 3(b) as the intercalated polymer mass $\left(m_{P}\right)$ normalized to the mass of the paper before intercalation $\left(m_{\mathrm{NT}}\right)$. For the low molecular weight polymer the mass uptake rises very rapidly before saturating after $1 \mathrm{~h}$ at $30-40 \%$ of the initial paper mass. However for the high molecular weight the increase is much more gradual, reaching $14 \%$ of the original paper mass after 360 hours with no sign of saturation.

As we know the specific surface area of the paper, $A_{S}$, it is possible to transform $\left(m_{P} / m_{\mathrm{NT}}\right)$ to represent the fraction of internal surface area covered by polymer, $\theta_{C}$. If we assume that the maximum coating thickness is one molecular layer then the fractional coverage can be expressed by

$$
\theta_{C}=\left(\frac{m_{P}}{m_{\mathrm{NT}}}\right) \frac{1}{A_{S} \rho_{P} h},
$$

where $\rho_{P}$ is the polymer density $\left(\rho_{P}=1660 \mathrm{~kg} / \mathrm{m}^{3}\right)$ and $h$ is the polymer film thickness, i.e., the molecular size. As we 


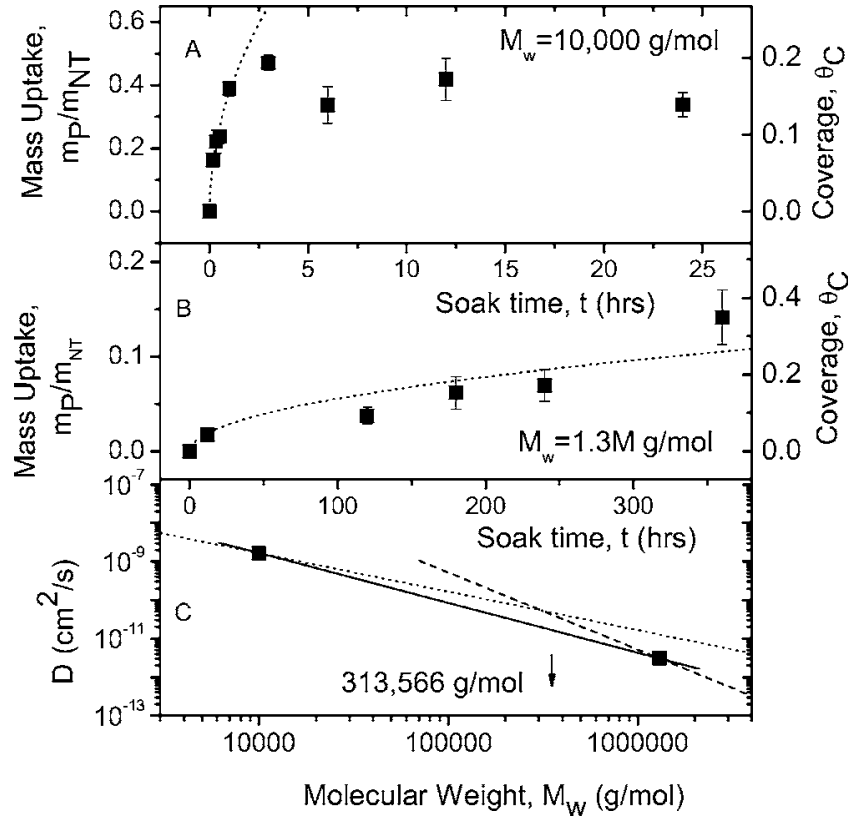

FIG. 3. (a) Polymer mass uptake as a function of soak time for PVP with $M_{w}=10000 \mathrm{~g} / \mathrm{mol}$. The dotted line is a fit to Eq. (2) with $D=1.66 \times 10^{-9} \mathrm{~cm}^{2} / \mathrm{s}$ and $l=60 \mu \mathrm{m}$. Note that the intercalation saturates after approximately $1 \mathrm{~h}$. (b) Polymer mass uptake as a function of soak time for PVP with $M_{w}=1.3 \mathrm{Mg} / \mathrm{mol}$. The dotted line is a fit to Eq. (2) with $D=3.08 \times 10^{-12} \mathrm{~cm}^{2} / \mathrm{s}$ and $l=60 \mu \mathrm{m}$. In this case there is no sign of saturation even after $360 \mathrm{~h}$. In both (a) and (b) the fraction of the internal surface coated by the polymer is shown as the right axis as calculated by Eq. (1). (c) Diffusion coefficients calculated from Figs. 3(a) and 3(b) as a function of $M_{w}$. The solid line is a fit to $D_{M} \propto M_{w}{ }^{-n}$, giving $n=1.29$. Rouse diffusion $(n=1)$ is depicted by the dotted line while reptation $(n=2)$ is illustrated by the dashed line. The intersection of these two regimes occurs at $M_{w}=313566 \mathrm{~g} / \mathrm{mol}$.

shall see below, it is very likely that the low $M_{w}$ polymer adsorbs as a random coil. The molecular size and hence the film thickness $(h)$ can be estimated as the root mean square radius of the coil $\left(R_{\mathrm{rms}}=\sqrt{ } 6 \times R_{g}\right),{ }^{14} R_{\mathrm{rms}}=2.4 \mathrm{~nm} \sim h$. Again, as we shall see later, the $M_{w}=1.3 \mathrm{Mg} / \mathrm{mol}$ polymer adsorbs in an extended state so we can estimate $h$ as close to the molecular thickness, i.e., slightly larger than the van der Waals distance, $h \sim 0.4 \mathrm{~nm}$. Using these values, and noting that the specific surface area of the pristine paper is 611 $\mathrm{m}^{2} / \mathrm{g}$, we can calculate the fractional surface coverage, $\theta_{C}$, as a function of soak time for both molecular weights. This is shown as the right axis in Fig. 3. We can clearly see that the mass uptake saturates for fractional coverage range 15$20 \%$ for the low $M_{w}$ polymer but is still increasing for fractional coverage of $35 \%$ for the high $M_{w}$ polymer.

The pores present in the Buckypaper can be considered as long highly branched channels of extremely irregular cross section. In general, diffusion can be characterized by an average displacement from the starting point, $\langle x\rangle$, that varies in time, $t$, as $\langle x\rangle \sim(D t)^{1 / 2}$ where $D$ is the diffusion coefficient. ${ }^{14}$ Similarly, Fickian mass transport of molecules into porous materials by diffusion displays similar temporal behavior with a square root time dependence. ${ }^{15}$ In this case, the inter- calated mass uptake as a function of time is given by ${ }^{16}$

$$
\frac{m_{P}}{m_{\mathrm{NT}}}=\left(\frac{m_{P}}{m_{\mathrm{NT}}}\right) \text { sat } \sqrt{\left(\frac{16 D}{b^{2} \pi}\right) t},
$$

where $\left(m_{P} / m_{\mathrm{NT}}\right)_{\text {sat }}$ is the saturated value of the mass uptake, $D$ is the diffusion coefficient, and $b$ is the film thickness.

Equation (2) was fitted to the data in Figs. 3(a) and 3(b) taking $b=60 \mu \mathrm{m}$. In both cases the fit was reasonably good, indicating the validity of Eq. (2). The fitting routine gave values of $D$ of $1.66 \times 10^{-9} \mathrm{~cm}^{2} / \mathrm{s}$ and $3.08 \times 10^{-12} \mathrm{~cm}^{2} / \mathrm{s}$ for the $10000 \mathrm{~g} / \mathrm{mol}$ and $1.3 \mathrm{Mg} / \mathrm{mol}$ polymers, respectively. These values are in the range expected for macromolecules confined within porous media. ${ }^{15}$ As one would imagine the diffusion constant for the higher molecular weight polymer is much lower compared to that for the $M_{w}=10000 \mathrm{~g} / \mathrm{mol}$ case.

The time scale for diffusion of a polymer in a porous medium depends on the size of the polymer molecule compared to the pore size, $d$. When the radius of gyration, $R_{g}$, of the polymer is much less than the pore size $\left(R_{g} \ll d\right)$ the polymer does not feel the strong topological constraints imposed by the pore walls and so can diffuse normally. Here the Rouse diffusion model is appropriate ${ }^{17-19}$ and $D \propto M_{w}{ }^{-1}$. In this situation the polymer retains its high entropy, randomly coiled state. However in the opposite regime when, $R_{g} \gg d$, the strong topological constraints imposed by the pore walls dominate and motion along the polymer's contour is much more favorable. This is known as reptation ${ }^{18-20}$ and is characterized by $D \propto M_{w}{ }^{-2}$ (Ref. 21).

The radius of gyration of a polymer chain is given by $R_{g}=l(N / 6)^{1 / 2}$, where $N$ is the number of repeat units and $l$ is the repeat unit length. ${ }^{14,18}$ Given that the length of the PVP repeat unit is $\sim 0.25 \mathrm{~nm}$ and its mass is $111.14 \mathrm{~g} / \mathrm{mol}$ we can calculate $R_{g}$ as $\sim 0.97 \mathrm{~nm}$ and $\sim 11.0 \mathrm{~nm}$ for the low and high molecular weight polymers, respectively. As the pore size distribution peaks at $4 \mathrm{~nm}$ (Fig. 2) we might expect the $M_{w}=10000 \mathrm{~g} / \mathrm{mol}$ polymer to diffuse according to the Rouse model and the $M_{w}=1.3 \mathrm{Mg} / \mathrm{mol}$ polymer to move by reptation. We can test this by plotting $D$ versus $M_{w}$ for both polymers and fitting to $D \propto M_{w}{ }^{-n}$. This is shown in Fig. 3(c) where the solid line represents the fit with $n=1.29$. The fact that $1<n<2$ suggests that the two molecular weights diffuse according to the different models as suggested above. The dependence of $D$ on $M_{w}$ is plotted as a dotted line for the Rouse model and as the dashed line for reptation. The crossover between these two regimes, as identified by the intersection of these lines, should occur for an $M_{w}$ corresponding to a polymer radius of gyration that matches the average pore size $\langle d\rangle .{ }^{19}$ This crossover occurs at $M_{w}=313566 \mathrm{~g} / \mathrm{mol}$ which corresponds to $R_{g}=5.4 \mathrm{~nm}$. This is in excellent agreement with the peak in the pore size distribution which occurs at $d=4 \mathrm{~nm}$.

This result is important as it means that the $M_{w}$ $=10000 \mathrm{~g} / \mathrm{mol}$ polymer diffuses through the Buckypaper as a randomly coiled chain. However, topological constraints mean that the $1.3 \mathrm{Mg} / \mathrm{mol}$ polymer is forced to unravel and move along its contour in an extended conformation. Furthermore when a given chain eventually adsorbs into the 


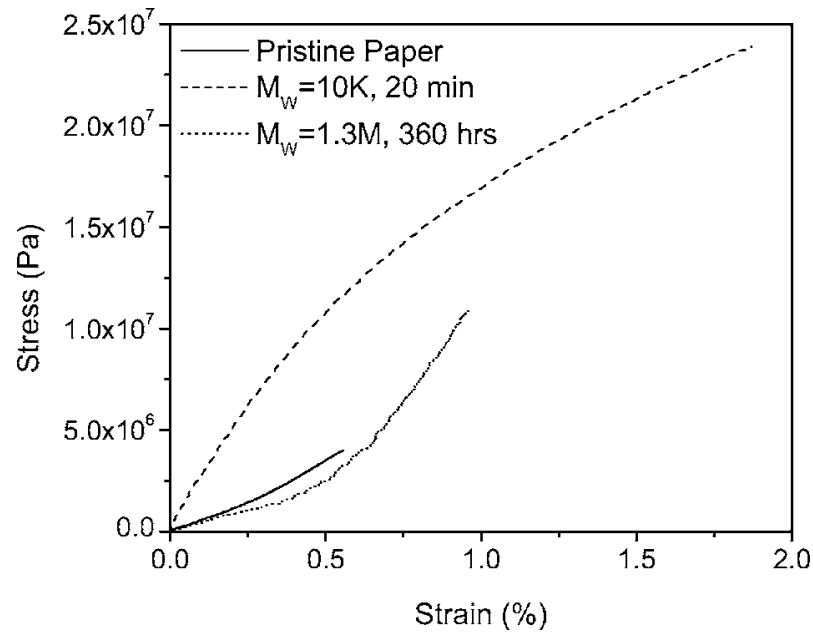

FIG. 4. Representative stress strain curves for the pristine paper, paper soaked in PVP with $M_{w}=10000 \mathrm{~g} / \mathrm{mol}$, and paper soaked in PVP with $M_{w}=1.3 M \mathrm{~g} / \mathrm{mol}$. Note that no individual stress strain curve displayed the average behavior for modulus, strength, and toughness. These curves were chosen to illustrate the general shape of the stress strain curves and their relative toughness'.

internal surface of the paper it is likely that it retains approximately its conformation while diffusing. This means that the intercalated polymer chains exist in very different confirmations for low and high molecular weight polymers. This is expected to have a significant effect on the physical properties of the polymer intercalated Buckypaper.

For both molecular weights, mechanical measurements, in the form of stress-strain curves were carried out as a function of mass uptake. Three typical curves for the pristine, annealed paper, paper soaked with $M_{w}=10000 \mathrm{~g} / \mathrm{mol}$ for 20 minutes and paper soaked with $M_{w}=1.3 M \mathrm{~g} / \mathrm{mol}$ for 360 hours are shown in Fig. 4. All curves displayed a linear region at low strain followed by plastic deformation above a strain of $\sim 0.3 \%$. Fracture was observed at strains of approximately $0.5 \%, 1.0 \%$, and $1.8 \%$ for the pristine paper, the $1.3 \mathrm{Mg} / \mathrm{mol}$ intercalated paper and the $10000 \mathrm{~g} / \mathrm{mol}$ intercalated papers, respectively. For all stress-strain curves the Young's modulus, $Y$ (slope at low strain), the ultimate tensile strength, $\sigma_{B}$, (stress at break), and toughness, $T$, (area under curve, energy per unit volume required to break) were calculated. These parameters are plotted for high and low molecular weights in Figs. 5 and 6, respectively, as a function of volume fraction, $V_{f}$. In all cases mass uptake was transformed into volume fraction using Eq. (3):

$$
V_{f}=\left[1+\left(\frac{\rho_{P}}{\rho_{\mathrm{NT}}}\right)\left(\frac{m_{\mathrm{NT}}}{m_{P}}\right)\right]^{-1},
$$

where $\rho_{P}$ and $\rho_{\mathrm{NT}}$ are the polymer and nanotube mass densities respectively $\left(\rho_{P}=1660 \mathrm{~kg} / \mathrm{m}^{3}, \rho_{\mathrm{NT}}=1,500 \mathrm{~kg} / \mathrm{m}^{3}\right)$. All the relevant parameters quoted in the next paragraph are given in Table I. (NB a different sheet of Buckypaper was used for each molecular weight intercalation study. Each sheet had slightly different mechanical properties. These are presented at the top of Table I.)

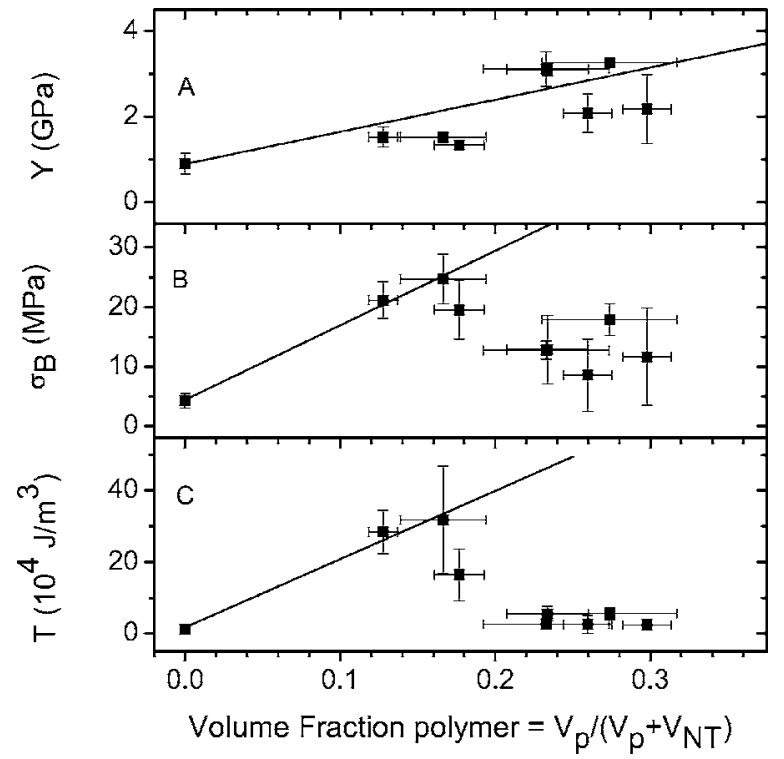

FIG. 5. Mechanical properties of Buckypaper intercalated with PVP of $M_{w}=10000 \mathrm{~g} / \mathrm{mol}$. (a) Young's modulus, $Y$, as a function of volume fraction. The line is a fit to Eq. (4) with $d Y / d V_{f}$ $=7.49 \mathrm{GPa}$. (b) Ultimate tensile strength, $\sigma_{B}$, as a function of volume fraction. At low volume fraction the data is linear with slope $d \sigma_{B} / d V_{f}=125 \mathrm{MPa}$. However, $\sigma_{B}$ tends to drop off above $V_{f}$ $=0.17$. (c) Toughness, $T$, as a function of volume fraction. At low volume fraction the data is linear with slope $d T / d V_{f}=1.91 \mathrm{MJ} / \mathrm{m}^{3}$. However, $T$ tends to drop off above $V_{f}=0.17$.

For the paper intercalated with the low molecular weight polymer (Fig. 5) the Young's modulus increases approximately linearly with a slope of $d Y / d V_{f}=7.49 \mathrm{GPa}$ before attaining a maximum value of $Y_{\max }=3.3 \mathrm{GPa}$ representing an increase over the pristine paper of $\times 3.6$. In comparison, the high molecular weight polymer (Fig. 6) displays a linear increase in Young's modulus $\left(d Y / d V_{f}=57.5 \mathrm{GPa}\right)$ at low volume fraction before saturating at $V_{f}=0.03$. The maximum modulus attained was $Y_{\max }=3.16 \mathrm{GPa}$ which was an increase of $\times 3.7$.

While the maximum values of Young's modulus attained are similar and agree with previous results ${ }^{6}$ the $d Y / d V_{f}$ values display a marked difference between molecular weights. Generally, the Young's modulus of composites are analyzed using the so-called rule of mixtures ${ }^{5,22}$ which states that for a mixture of components 1 and 2 , the resultant modulus, $Y$, is given by

$$
Y=\left(Y_{1}-Y_{2}\right) V_{f}+Y_{2},
$$

where $V_{f}$ is the volume fraction of phase 1 . In our case, treating the polymer as phase 1 , and using equation (4), the measured $d Y / d V_{f}$ values give polymer moduli of $8.4 \mathrm{GPa}$ and 58.4 GPa for the low and high molecular weight polymers, respectively. These values are much too large to be realistic as typical non-conjugated polymers such as poly(vinylalcohol) and poly(styrene) have moduli in the $0.2-4 \mathrm{GPa}$ range. ${ }^{23}$ The fact that these values are too large suggests that the reinforcement mechanism is not one of typical mixing. 


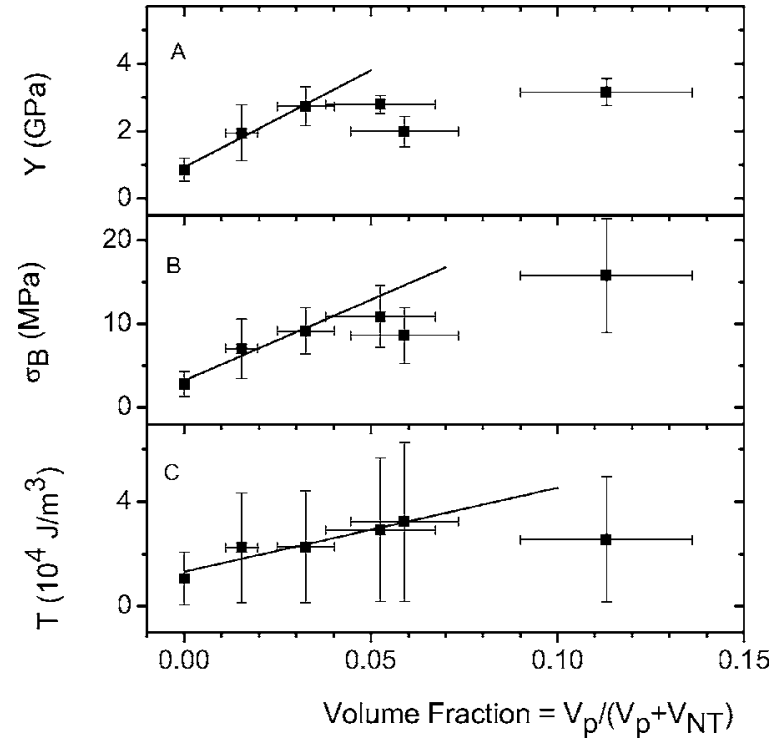

FIG. 6. Mechanical properties of Buckypaper intercalated with PVP of $M_{w}=1.3 \mathrm{Mg} / \mathrm{mol}$. (a) Young's modulus, $Y$, as a function of volume fraction. The line is a fit to Eq. (4) with $d Y / d V_{f}$ $=57.5 \mathrm{GPa}$. However, $Y$ tends to saturate above $V_{f}=0.04$. (b) Ultimate tensile strength, $\sigma_{B}$, as a function of volume fraction. At low volume fraction the data is linear with slope $d \sigma_{B} / d V_{f}=193 \mathrm{MPa}$. However, $\sigma_{B}$ tends to saturate above $V_{f}=0.04$. (c) Toughness, $T$, as a function of volume fraction. At low volume fraction the data is linear with slope $d t / d V_{f}=321 \mathrm{~kJ} / \mathrm{m}^{3}$. However, $T$ tends to saturate above $V_{f}=0.04$.

In the case of ultimate tensile strength the picture is similar. For the paper intercalated with the low molecular weight polymer (Fig. 5) the strength increases approximately linearly with a slope of $d \sigma_{B} / d V_{f}=125 \mathrm{MPa}$ before reaching a maximum value of $\sigma_{B, \max }=24.7 \mathrm{MPa}$ (increase: $\times 5.8$ ) at a volume fraction of 0.17 . Above this volume fraction the strength drops off. In comparison, the high molecular weight polymer (Fig. 6) also displays a linear increase in strength $\left(d \sigma_{B} / d V_{f}=193 \mathrm{MPa}\right)$ at low volume fraction before saturating at $V_{f}=0.03$. The maximum strength attained was $\sigma_{B, \max }$ $=15.8 \mathrm{MPa}$ which represents an increase of $\times 5.6$. Again, in both cases the increase in strength compared to the pristine Buckypaper was similar while $d \sigma_{B} / d V_{f}$ was significantly larger for the higher molecular weight polymer. As in the case with the modulus, these results cannot be explained by the intrinsic strength of the polymers themselves.

Finally, the toughness results are in contrast to those for the modulus and strength. For the paper intercalated with the low molecular weight polymer (Fig. 5) the toughness also increases approximately linearly with a slope of $d T / d V_{f}$ $=191 \mathrm{~J} / \mathrm{m}^{3}$ before reaching a maximum value of $T=31.8$ $\times 10^{4} \mathrm{~J} / \mathrm{m}^{3}$ (increase: $\times 26$ ) at a volume fraction of 0.17 . Above this volume fraction the toughness drops off. However, the high molecular weight polymer (Fig. 6) also displays a linear increase in toughness but with a much lower slope $\left(d T / d V_{f}=32.1 \mathrm{~J} / \mathrm{m}^{3}\right)$ at low volume fraction before saturating at $V_{f}=0.03$. In this case the maximum toughness was only $T=3.23 \times 10^{4} \mathrm{~J} / \mathrm{m}^{3}$ which represents an increase of only $\times 3.0$. This raises the question: why do strength and
TABLE I. Summary of values found in this study. Comparisons between absolute values of the maximum in mechanical properties may be misleading as a different sheet of Buckypaper was used for each molecular weight intercalation study. Each sheet had slightly different properties. These are presented at the top of the table. A more reliable value is the maximum relative increase in properties. This is shown in brackets.

\begin{tabular}{lll}
\hline \hline & $\begin{array}{l}M_{w}=10000 \\
\mathrm{~g} / \mathrm{mol}\end{array}$ & $\begin{array}{l}M_{w}=1.3 M \\
\mathrm{~g} / \mathrm{mol}\end{array}$ \\
\hline$Y_{\mathrm{NT}}(\mathrm{GPa})$ & 0.903 & 0.857 \\
$\sigma_{\mathrm{NT}}(\mathrm{MPa})$ & 4.29 & 2.8 \\
$T_{\mathrm{NT}}\left(\mathrm{J} / \mathrm{m}^{3}\right)$ & 1.22 & 1.06 \\
$\left(m_{P} / m_{\mathrm{NT}}\right)_{\max }$ & 0.47 & 0.14 \\
$D\left(\mathrm{~cm}^{2} / \mathrm{s}\right)$ & $1.66 \times 10^{-9}$ & $3.08 \times 10^{-12}$ \\
$Y_{C, \max }(\mathrm{GPa})$ & $3.26(\times 3.6)$ & $3.16(\times 3.7)$ \\
$d Y_{C} / d V_{f}$ & 7.49 & 57.4 \\
$\sigma_{C, \max }(\mathrm{MPa})$ & $24.7(\times 5.8)$ & $15.8(\times 5.6)$ \\
$d \sigma_{C} / d V_{f}(\mathrm{MPa})$ & 125 & 193 \\
$T_{C, \max }\left(10^{4} \times \mathrm{J} / \mathrm{m}^{3}\right)$ & $31.8(\times 26)$ & $3.23(\times 3.0)$ \\
$d T_{C} / d V_{f}\left(\mathrm{~J} / \mathrm{m}^{3}\right)$ & 191 & 32.1 \\
\hline \hline
\end{tabular}

modulus increase more efficiently for the high $M_{w}$ intercalant but toughness displays the opposite behavior?

This question can be answered by examining both the fractional surface coverage and conformations of the two types of intercalated polymers. As shown in Figs. 5 and 6, both strength and toughness saturate at volume fractions, $V_{f}$ $\left(m_{P} / m_{\mathrm{NT}}\right)$ of $0.17(0.23)$ and $0.03(0.037)$ for the low and high molecular weights, respectively. Using Eq. (1), we can calculate the fractional surface coverage, $\theta_{C}$, as $\sim 9 \%$ for both molecular weights at saturation. Thus taking the conformation into account, the fractional surface coverage over the linear regime is similar for both polymers. Thus variations in fractional surface coverage cannot explain the differences in mechanical properties.

This means that the differences must lie in the polymer conformations rather than in surface coverage. We have shown that the mechanism of reinforcement is not that of simple mixing. One other possibility is that the intercalated polymer acts as a binder which enhances the interbundle adhesion as suggested previously. ${ }^{6}$ As noted above, the low $M_{w}$ polymer adsorbs in the coiled conformation. This would tend to act as a rather localized, inefficient binder, connecting no more than two bundles per molecule. In contrast the high molecular weight polymer adsorbs in an extended conformation which has a contour length of approximately $3 \mu \mathrm{m}$ ( $=N l)$. These extended strands act to bind a large number of bundles together acting as a very efficient binder. This would result in more efficient enhancement of both strength and modulus for the paper intercalated with the high molecular weight polymer.

This model also explains why the toughness is higher for the low $M_{w}$ material. When a stress is applied, a large amount of work must be done to unravel and finally separate two bundles initially bound by a low $M_{w}$, randomly coiled strand. This is because it requires a significant energy input 
to go from a high entropy, coiled state to a low entropy, extended state. This results in a relatively high toughness. This is a similar mechanism to that which gives rubber its elasticity. ${ }^{24}$ However for the high $M_{w}$ polymer, as it is already extended, there is little scope to unravel it on application of a stress resulting in lower toughness.

We can very crudely estimate the potential of this method for toughness enhancement. The contribution to the toughness per polymer strand from the work required to unravel the coil is given by the free energy increase, $\Delta G=-T \Delta S$, where $\Delta S$ is the increase in entropy associated with going from a coiled to an extended state. This can be approximated, for a uniaxial force by ${ }^{25}$

$$
\Delta S \sim-\frac{k}{2} \lambda^{2},
$$

where $k$ is Boltzmann's constant and $\lambda$ is the chain extension ratio. This can be approximated as the ratio of the chain end to end separation before extension to that after. The average end-to-end separation for a random coil is $R_{\mathrm{rms}}=l \sqrt{ } N$ (the symbols have the same meaning as before). If we assume complete extension, then the end-to-end length (contour length) afterwards is $\sim N l$. This means that $\lambda=\sqrt{ } N$, giving $\Delta G \sim \frac{1}{2} k T N$ per chain. The number of chains per volume is

$$
N_{\text {chain }} / V=\left(\frac{m_{P}}{m_{\mathrm{NT}}}\right) \frac{\rho_{\text {paper }}}{m_{\text {chain }}},
$$

where $m_{\text {chain }}$ is the mass of each polymer chain. This means that the toughness (or work done per unit volume) can be expressed as

$$
T=\frac{k T N}{2}\left(\frac{m_{P}}{m_{\mathrm{NT}}}\right) \frac{\rho_{\text {paper }}}{m_{\text {chain }}} .
$$

For the $10000 \mathrm{~g} / \mathrm{mol}$ polymer at saturation $\left(m_{P} / m_{\mathrm{NT}}\right.$ $=0.23$ ) this yields a (calculated) toughness of $T=1.33$ $\times 10^{6} \mathrm{~J} / \mathrm{m}^{3}$. This is within order of magnitude agreement with the measured toughness of $T \sim 3 \times 10^{5} \mathrm{~J} / \mathrm{m}^{3}$. We would expect the calculated value to be greater than the measured value as it is unlikely that the chains would fully unravel before film fracture. However, the agreement of even such a crude estimate with the measured value is encouraging and suggests that toughness can be tailored by control of the molecular weight. Indeed, the toughness could be maximized by intercalating the maximum possible molecular weight polymer that can still diffuse as a random coil. In the case of the Buckypaper used here, that would be for $M_{w}$ $\sim 313566 \mathrm{~g} / \mathrm{mol}\left(R_{g} \sim\langle d\rangle\right)$.

This result is important as fibers fabricated from SWNT and poly(vinylalcohol) have been shown to be very strong and more importantly, among the toughest macro scale materials known to man. ${ }^{3}$ However, the molecular weight of the polymer used for fiber production has yet to be optimized. In our work we have shown that in certain circumstances where polymer conformation is constrained during sample preparation, molecular weight can have a large bearing on the resultant mechanical properties. While high molecular weight polymer binders are important for strength enhancement, shorter chains can be used to enhance toughness. This would be particularly useful for example, as a postproduction treatment for directly spun nanotube fibers. ${ }^{26,27}$ We believe that careful choice of molecular weight or even blending of two or more polymers of different molecular weights could result in further improvements in these already impressive fiber materials.

\section{CONCLUSION}

In conclusion we have shown that SWNT Buckypaper contains a significant fraction $(60-70 \%)$ of free volume in the form of one-dimensional branched pores. These pores are characterized by a diameter distribution that varies from one to well over a hundred nanometers. An amphiphillic, nonconjugated polymer, such as PVP, could easily be intercalated into these pores simply by soaking the Buckypaper in polymer solutions at both low and very high molecular weights. By monitoring the intercalated mass uptake as a function of time, the polymer diffusion coefficient could be calculated for each molecular weight. Comparison of the diffusion coefficients as a function of $M_{w}$ suggested that the low weight polymer diffuses normally through the pores while the larger polymer moves by reptation. This is significant as it means that the low and high molecular weight strands move as a random coil and in an extended state, respectively. As it is likely that this conformation will be, at least partially, retained on adsorption this will be significant for the properties of the resultant composite. Mechanical measurements show that Young's modulus, strength and toughness of the Buckypaper are all enhanced by polymer intercalation. However, enhancement of both modulus and strength is significantly more efficient for papers intercalated with the high molecular weight polymer. In contrast the opposite scenario is observed for toughness. This apparent paradox in mechanical properties can be explained by differences in polymer conformation ("coil" for low $M_{w}$ and "extended chain" for high $M_{w}$ polymer) and their ability to bind and unravel under applied stress. This underlines the importance of polymer confirmation for reinforcement in nanotube containing composites. This result could be used to significantly enhance the properties of state-of-the-art polymer-nanotube fibers.

\section{ACKNOWLEDGMENTS}

M.i.h.P. gratefully acknowledges financial support from the University of Texas at Dallas. C.J.F., A.I.M., W.J.B., and J.N.C. would like to thank the Irish Higher Education Authority and Science Foundation Ireland. 
*Author to whom correspondence should be addressed. Electronic address: M.Panhuis@hull.ac.uk

${ }^{1}$ D. A. Walters, L. M. Ericson, M. J. Casavant, J. Liu, D. T. Colbert, K. A. Smith, and R. E. Smalley, Appl. Phys. Lett. 74, 3803 (1999).

${ }^{2}$ M.-F. Yu, B. S. Files, S. Arepalli, and R. S. Ruoff, Phys. Rev. Lett. 84, 5552 (2000).

${ }^{3}$ A. B. Dalton, S. Collins, E. Munoz, J. M. Razal, V. H. Ebron, J. P. Ferraris, J. N. Coleman, B. G. Kim, and R. H. Baughman, Nature (London) 423, 703 (2003).

${ }^{4}$ M. Cadek, J. N. Coleman, K. P. Ryan, V. Nicolosi, G. Bister, A. Fonseca, J. B. Nagy, K. Szostak, F. Béguin, and W. J. Blau, Nano Lett. 4, 353 (2004).

${ }^{5}$ J. N. Coleman, M. Cadek, R. Blake, V. Nicolosi, K. P. Ryan, C. Belton, A. Fonseca, J. B. Nagy, Y. K. Gun'ko, and W. J. Blau, Adv. Funct. Mater. 14, 791 (2004).

${ }^{6}$ J. N. Coleman, W. J. Blau, A. B. Dalton, E. Munoz, S. Collins, B. G. Kim, J. Razal, M. Selvidge, G. Viero, and R. H. Baughman, Appl. Phys. Lett. 82, 1682 (2003).

${ }^{7}$ P. Nikolaev, M. J. Bronikowski, R. Kelley Bradley, F. Rohmund, D. T. Colbert, K. A. Smith, and R. E. Smalley, Chem. Phys. Lett. 313, 91 (1999).

${ }^{8}$ G. Horvath and K. Kawazoe, J. Chem. Eng. Jpn. 16, 470 (1983).

${ }^{9}$ E. P. Barret, L. G. Joyner, and P. H. Halenda, J. Am. Chem. Soc. 73, 373 (1951).

${ }^{10}$ Pore diameter, $D_{\text {pore }} \sim\left[R^{2}\{4 \sqrt{ } 3 / \pi(1+d / R)-2 / \pi\}+\sqrt{ } 3 / \pi d^{2}\right]^{1 / 2}$, where $R$ is the nanotube radius and $d$ is the van der Waals distance.

${ }^{11}$ W. Zhou, Y. H. Ooi, R. Russo, P. Papanek, D. E. Luzzi, J. E. Fischer, M. J. Bronikowski, P. A. Willis, and R. E. Smalley, Chem. Phys. Lett. 350, 6 (2001).
${ }^{12}$ S. Brunauer, P. H. Emmett, and E. Teller, J. Am. Chem. Soc. 60, 309 (1938).

${ }^{13}$ S. M. Cooper, H. F. Chuang, M. Cinke, B. A. Cruden, and M. Meyyappan, Nano Lett. 3, 189 (2003).

${ }^{14}$ P. W. Atkins, Physical Chemistry (Oxford University Press, New York, 1990).

${ }^{15}$ A. T. Fiory, S. Martin, L. F. Schneemeyer, R. M. Fleming, A. E. White, and J. V. Waszczak, Phys. Rev. B 38, 7129 (1988).

${ }^{16}$ N. Vahdat and V. D. Sullivan, J. Appl. Polym. Sci. 79, 1265 (2001).

${ }^{17}$ P. E. Rouse, J. Chem. Phys. 21, 1272 (1953).

${ }^{18} \mathrm{M}$. Doi and S. F. Edwards, The Theory of Polymer Dynamics (Clarendon Press, Oxford, 1986).

${ }^{19}$ S. E. Lee and A. K. Chakraborty, J. Chem. Phys. 117, 23 (2002).

${ }^{20}$ P. G. de Gennes, J. Chem. Phys. 55, 572 (1971).

${ }^{21}$ In the intermediate case another mode characterized by entropic trapping may contribute; however, we will not consider this here; see J. Rousseau, G. Drouin, and G. W. Slater, Phys. Rev. Lett. 79, 10 (1997).

${ }^{22}$ H. Krenchel, Fibre Reinforcement (Akedemisk Forlag, Copenhagen, 1964)

${ }^{23}$ W. D. Callister, Materials Science and Engineering, an Introduction (Wiley, New York, 2003).

${ }^{24}$ H. Pelzer, Rep. Prog. Phys. 6, 330 (1939).

${ }^{25}$ S. F. Sun, Physical Chemistry of Macromolecules (Wiley, New York, 2004).

${ }^{26}$ Y. L. Li, I. A. Kinloch, and A. H. Windle, Science 304, 276 (2004).

${ }^{27}$ M. Zhang, K. R. Atkinson, and R. H. Baughman, Science 306, 1358 (2004). 